\title{
Development of a new type of inflatable structure - the adaptive rescue cushion
}

\author{
Rami Faraj*, Blażej Poplawski*, Dorian Gabryel*, Tomasz Kowalski* and Krzysztof Hinc* \\ * Institute of Fundamental Technological Research Polish Academy of Sciences \\ Pawinskiego 5B, 02-106 Warszawa, Poland \\ e-mail: rfaraj@ippt.pan.pl, web page: http://www.ippt.pan.pl
}

\begin{abstract}
The contribution concerns a special type of an inflatable structure, which can be classified within the group of airbag systems. Airbags are often applied in different types of protective structures and systems, their most common application is crash protection of people [1]. Besides automotive industry the airbags are considered for such systems as offshore turbines [2], unmanned aerial vehicles [3] or even Martian landers [4]. Another impressive application of them and simultaneously the main subject of this contribution is evacuation of people from heights. In such application airbags are used as socalled rescue cushions, which are operated by fire brigades in case the conventional evacuation is unavailable. Although relatively high number of patents can be found, the scientific literature in this field is very limited. Nevertheless, importance of the problem and appearing accidents motivated the authors to start the research on development of a new, safer type of rescue cushion system, which will guarantee high performance and adaptive capabilities. The preliminary study [5] revealed possibility of significant improvement of the system response for a range of excitation conditions. Further research is conducted in order to provide a solution ensuring successful adaptation of the rescue cushion to the evacuation height, as well as the mass of landing person. The general design methodology and objective of optimization are presented within exemplary case study. Constraints resulting from legal, functional and economic requirements are discussed in detail. Main challenges and directions of further research are indicated.
\end{abstract}

\section{REFERENCES}

[1] J. Richert, D. Coutellier, C. Götz et al., Advanced smart airbags: The solution for real-life safety?, Int. J. Crashworthiness, 12, 159-171 (2007).

[2] C. Graczykowski and J. Holnicki-Szulc, Protecting offshore wind turbines against ship impacts by means of Adaptive inflatable Structures, Shock. Vib., 16(4), 335-353 (2009).

[3] Z. Wołejsza, J. Holnicki-Szulc, C. Graczykowski et al., Dynamics and control of adaptive airbags for UAV impact protection, ISMA 2018 / USD 2018, 17-19/09/2018, Leuven, Belgium, pp. 36613670 (2018).

[4] P. Cao, X. Hou, Y. Wang, L. Meng, R. Xiaoshan, Y. Shi, Flexible airbag cushioning for Martian landing based on discrete element method, Adv. Space Res., 63(8), 2566-2583 (2019).

[5] R. Faraj, B. Popławski, K. Hinc, Preliminary study on modelling and optimization of the rescue cushion system, ISMA2020 / USD2020, 07-09/09/2020, Leuven, Belgium, 2675-2684, (2020). 